\title{
Characterization of pneumonia and other factors leading to poorer survival across all age groups in patients with non-small cell lung cancer (NSCLC)
}

\author{
Frank D. Weinberg ${ }^{1,2 \# *}$, Lili Zhao ${ }^{3 \#}$, Niranjana Chellappa ${ }^{4}$, Gregory P. Kalemkerian ${ }^{1}$, Nithya Ramnath ${ }^{1,2}$ \\ ${ }^{1}$ Division of Hematology/Oncology, Department of Internal Medicine, University of Michigan, MI, USA; ${ }^{2}$ VA Ann Arbor Health Care System, \\ Ann Arbor, MI, USA; ${ }^{3}$ Department of Biostatistics, Ann Arbor, MI, USA; ${ }^{4}$ Department of Medicine, St. Joseph Mercy Ann Arbor Hospital, \\ Ypsilanti, MI, USA \\ Contributions: (I) Conception and design: FD Weinberg, N Ramnath, L Zhao; (II) Administrative support: FD Weinberg; (III) Provision of study \\ materials or patients: FD Weinberg, N Ramnath; (IV) Collection and assembly of data: FD Weinberg, L Zhao, N Chellappa; (V) Data analysis and \\ interpretation: All authors; (VI) Manuscript writing: All authors; (VII) Final approval of manuscript: All authors. \\ \#These authors contributed equally to this work. \\ Correspondence to: Frank D. Weinberg. University of Michigan, 300 N Ingalls St., N13A23 N Ingalls Bldg, SPC 5419, Ann Arbor, MI 48109, USA. \\ Email: fweinber@umich.edu.
}

Background: Lung cancer death rates and incidence in both men and women have decreased over the past two decades. However, certain subsets of non-small cell lung cancer (NSCLC) have arisen with poor outcomes. Identifying factors which contribute to poorer outcomes as well as those that inform early detection strategies remain unmet needs. We present data from a contemporaneous group of NSCLC patients that received care at a single University teaching hospital to understand clinical and pathological factors influencing outcomes in the past decade.

Methods: A cohort of 2,289 patients with NSCLC who established care at the Rogel Cancer Center, University of Michigan between January 2011 and April 2019 were identified. Patient characteristics and clinical outcomes were recorded using electronic health records. The Kaplan-Meier method and the Cox proportional model were used to assess relationship between clinic-pathological factors and survival.

Results: Of the 2,289 patients, $92 \%$ were $>50$ years of age while $8 \%$ were $<50$ years of age. The majority $(70 \%)$ of older patients were former smokers. The majority (61\%) of younger patients were diagnosed as having Stage IV NSCLC. Among younger patients, $87 \%$ had histologically confirmed non-squamous histology. Univariate analysis revealed that overall survival was significantly lower in patients diagnosed with pneumonia prior to the diagnosis of NSCLC than in those who were not diagnosed with pneumonia (1.9 vs. 21.8 months; $\mathrm{P}<0.001$ ). Multivariate analysis revealed that older patients had poorer survival than younger patients (HR 1.57, 95\% CI: 1.10-2.06, $\mathrm{P}=0.01$ ) and that patients diagnosed with pneumonia prior to their lung cancer diagnosis had poorer survival across all age groups, particularly in those with advanced-stage disease.

Conclusions: Findings from this study merit prospective studies to understand cost-benefit ratio of followup cross sectional imaging of all patients diagnosed with unprovoked pneumonia, including in younger non/ current smokers.

Keywords: Non-small cell lung cancer (NSCLC); pneumonia, survival

Submitted Sep 14, 2020. Accepted for publication Dec 03, 2020.

doi: $10.21037 /$ jtd-20-2891

View this article at: http://dx.doi.org/10.21037/jtd-20-2891

\footnotetext{
*After 2/16/2021, Dr. Frank D. Weinberg will be affiliated with University of Illinois Health System and will have email fweinb1@uic.edu.
} 


\section{Introduction}

Lung cancer is the leading cause of cancer-related death in the United States. Despite advances in lung cancer treatments over the past decade, the 5-year survival remains below $20 \%$ in patients with non-small cell lung cancer (NSCLC). Since the initiation of tobacco-control campaigns, there has been a steady decrease in overall lung cancer incidence and mortality (1). Since 1990, the lung cancer death rate has declined by $51 \%$ in males and $26 \%$ in females in the United States (2). Despite the overall reduction in lung cancer incidence, a number of studies have demonstrated increased incidence in certain subgroups of patients, especially in young women and never smokers (3-7). However, other studies have reported that these subgroups may not have increased lung cancer incidence $(5,8,9)$.

The median age at diagnosis of NSCLC is 70 years and less than $5 \%$ of patients are younger than 50 . Studies have suggested that there are potential differences in factors such as sex, smoking history, family history of lung cancer, histology and stage at diagnosis between young and old patients with conflicting data regarding outcomes (10-17). Given the shifting demographics and differences in subgroups of NSCLC patients, it is imperative to understand clinical prognostic factors among the various age groups over the past decade. Therefore, we set out to study clinical and pathological factors of NSCLC in a contemporaneous set of patients treated at a major teaching hospital in the United States. The aims of the current study were to (I) identify key differences in demographics between older and younger patients with lung cancer and assess their overall survival and (II) determine potential underlying factors that may contribute to poorer outcomes. We present the following article in accordance with the STROBE reporting checklist (available at http://dx.doi.org/10.21037/jtd-20-2891).

\section{Methods}

\section{Patients}

Patients who were diagnosed and treated for NSCLC at the Rogel Cancer Center of the University of Michigan were identified retrospectively between January 2011 and April 2019. Patients were sorted by age at diagnosis and divided into two cohorts; 50 years of age or younger or older than 50 years of age. Previous studies have generally defined younger patients as those less than 50 years of age $(10,18-22)$. Smokers were classified as current (if smoking at time of diagnosis), former (someone who has smoked at least 100 cigarettes, but quit at the time of diagnosis) or never smokers. Diagnosis was based on ICD codes which were confirmed with tumor pathology via surgical or biopsy specimen or cytology examination. Exclusion criteria included patients with small cell lung cancer. The study was conducted in accordance with the Declaration of Helsinki (as revised in 2013). The study was approved by the University of Michigan Institutional Review Board (IRB) (HUM00125235) and individual consent for this retrospective analysis was waived.

\section{Data collection}

Medical records were reviewed retrospectively using the University of Michigan Data Direct Database. This database allows access to clinical data from more than 4 million unique patients from across the University of Michigan Health System enterprise. Patient demographics, smoking history, date of diagnosis, histology, stage at diagnosis, date of encounters for pneumonia, and survival data were collected. Earliest date of diagnosis of pneumonia was collected and compared to NSCLC diagnosis date based on ICD-10 coding for pneumonia (J18.9).

\section{Statistical analysis}

Two-sample t tests were used to compare continuous variables between young and old patients and Chi-squared/ Fisher's exact tests were used to compare categorical variables between young and old patients. Overall patient survival was measured from the date of diagnosis to death due to any reason. Data were censored at the last followup or 5 years, whichever occurred first, for patients who were alive at the time of analysis. Survival curves were constructed using the method of Kaplan-Meier and survival differences were assessed using the log-rank test. To appropriately account for potential confounders, Cox proportional hazards model was used. Final Cox model included pneumonia, age, gender, race, stage, smoking status, and an interaction between pneumonia and stage (interactions between pneumonia and other covariates were not significant and, therefore were not included in the final model). Martingale-Based residuals were used to check the proportional hazard assumption in the Cox model. Statistical significance was defined as a $\mathrm{P}$ value $<0.05$. All analyses were performed using SAS 9.4 software. 
Table 1 Patient demographic characteristics

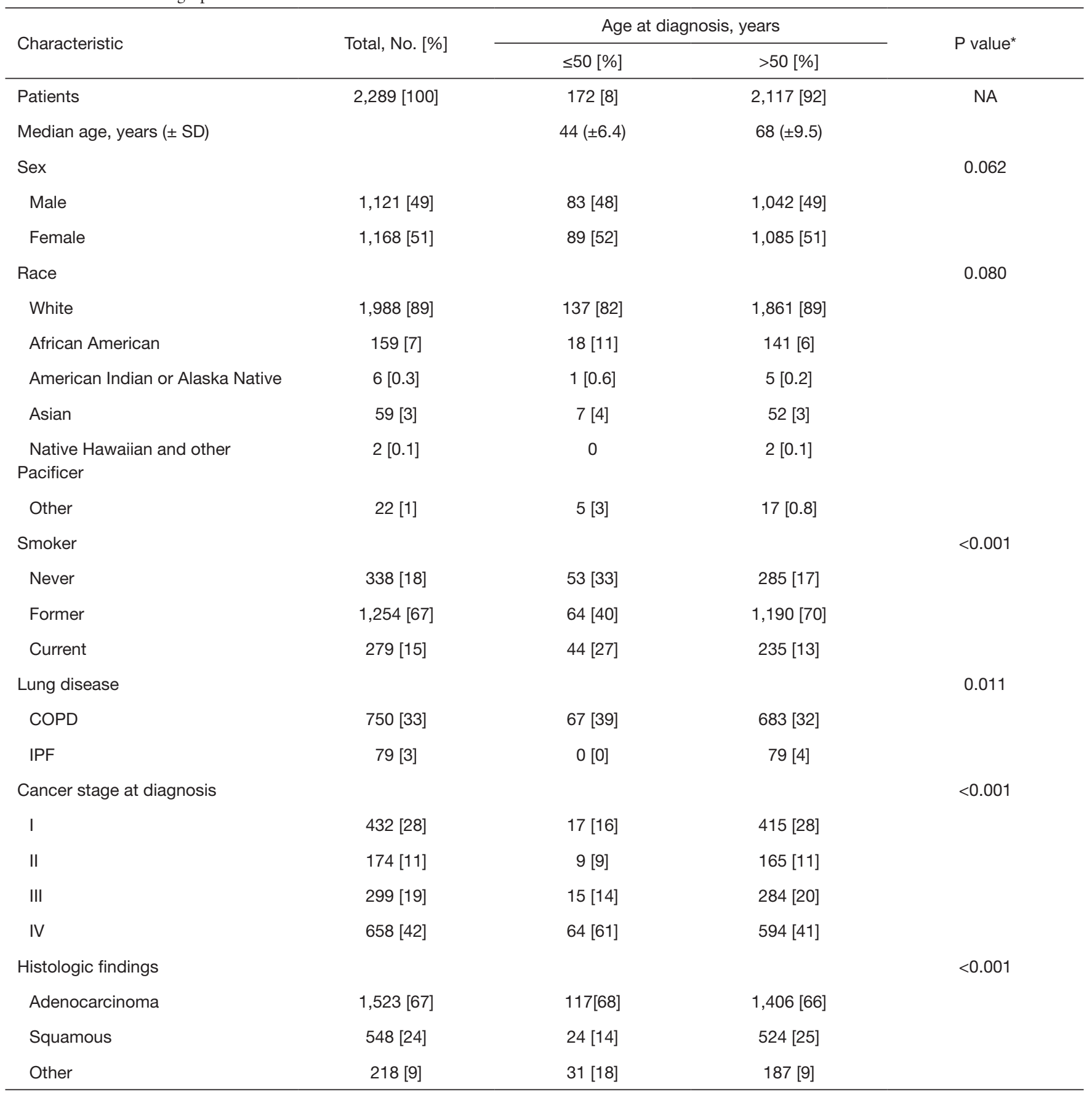

${ }^{*} \mathrm{P}$ value based on the difference in the proportional incidence distribution between younger and older patient populations for each parameter.

\section{Results}

\section{Demographic characteristics}

Between January 2011 and April 2019, 172 patients were diagnosed with NSCLC at age 50 years or younger, accounting for $8 \%$ of all $(\mathrm{n}=2,289)$ newly diagnosed NSCLC patients. The median age was 44 years $(\mathrm{SD} \pm 6.4$ years) in the younger cohort and 68 years $(\mathrm{SD} \pm 9.5$ years) in the older cohort. The female-to-male distribution was similar in both age cohorts (Table 1). The majority of patients in 
Table 2 Univariate survival analysis

\begin{tabular}{lcc}
\hline Predictors & HR $(95 \%$ Cl) & P value \\
\hline Age (old vs. young) & $1.10(0.89,1.36)$ & 0.38 \\
Gender (female vs. male) & $0.85(0.76,0.95)$ & 0.005 \\
Race (white vs. non-white) & $1.19(0.99,1.42)$ & 0.06 \\
Histology & & $<0.0001$ \\
Squamous vs. adenocarcinoma & $1.16(1.02,1.32)$ & \\
Other vs. adenocarcinoma & $1.90(1.60,2.25)$ & \\
Smoking status & & 0.19 \\
Current vs. never & $1.22(0.99,1.51)$ & \\
Former vs. never & $1.10(0.93,1.30)$ & \\
Pneumonia & & \\
Prior vs. after cancer diagnosis & $2.38(1.75,3.33)$ & \\
Prior vs. no cancer diagnosis & $2.94(2.17,4.00)$ & \\
Stage & & \\
III vs. I/II & $2.41(1.93,3.01)$ & \\
IV vs. I/II & $5.15(4.28,6.19)$ & \\
\hline
\end{tabular}

younger and older cohorts were Caucasian ( $82 \%$ vs. 89\%) which accurately reflects the overall patient demographics at Michigan Medicine. The younger cohort had fewer former ( $40 \%$ vs. $70 \%$ ), but more never smokers ( $33 \%$ vs. $17.0 \%$ ) and current smokers (27\% vs. $13 \%)$ than the older cohort (Table 1). Additionally, the younger cohort had an increased number of COPD diagnoses as compared to the older cohort (39\% vs. $23 \%)$.

\section{Tumor characteristics}

Younger patients were more likely to have stage IV NSCLC at diagnosis than older patients (61\% vs. $41 \%)$ and less likely to have stage I disease (16\% vs. $28 \%$ ). While there was not a significant difference in adenocarcinoma diagnoses between older and younger patients, there were more older patients diagnosed with squamous cell carcinoma while conversely, there were more younger patients diagnosed with histologic subtypes other than adenocarcinoma and squamous cell carcinoma (Table 1).

\section{Survival}

Univariate analysis demonstrated that male gender, non-
Table 3 Multivariate analysis for survival

\begin{tabular}{|c|c|c|}
\hline Predictors & $\mathrm{HR}(95 \% \mathrm{Cl})$ & $P$ value \\
\hline Age (old vs. young) & $1.506(1.10,2.06)$ & 0.01 \\
\hline Gender (female vs. male) & $0.843(0.719,0.988)$ & 0.035 \\
\hline Race (white vs. non-white) & $1.138(0.89,1.46)$ & 0.30 \\
\hline Histology & & $<0.0001$ \\
\hline Other versus adenocarcinoma & $1.816(1.43,2.31)$ & \\
\hline $\begin{array}{l}\text { Squamous versus } \\
\text { adenocarcinoma }\end{array}$ & $1.42(1.17,1.74)$ & \\
\hline $\begin{array}{l}\text { Pneumonia and histology } \\
\text { interaction }\end{array}$ & & 0.58 \\
\hline Smoking status & & 0.79 \\
\hline Current vs. never & $1.087(0.83,1.42)$ & \\
\hline Former vs. never & $1.068(0.87,1.32)$ & \\
\hline $\begin{array}{l}\text { Pneumonia and stage } \\
\text { interaction }\end{array}$ & & 0.0072 \\
\hline \multicolumn{3}{|l|}{ Stage I/II } \\
\hline Prior vs. no pneumonia & $1.32(0.49,3.48)$ & \\
\hline Prior vs. after pneumonia & $0.56(0.21,1.49)$ & \\
\hline \multicolumn{3}{|l|}{ Stage III } \\
\hline Prior vs. no pneumonia & $3.54(1.49,8.40)$ & \\
\hline Prior vs. after pneumonia & $3.27(1.37,7.75)$ & \\
\hline \multicolumn{3}{|l|}{ Stage IV } \\
\hline Prior vs. no pneumonia & $4.03(2.74,5.95)$ & \\
\hline Prior vs. after pneumonia & $2.87(1.92,4.27)$ & \\
\hline
\end{tabular}

adenocarcinoma histology, advanced stage and pneumonia prior to lung cancer diagnosis were poor prognostic factors. There was no significant difference between younger and older patients (21 vs. 19.4 months; $\mathrm{P}=0.38$ ) (Table 2). However, multivariate analysis revealed that older patients had a poorer median survival than younger patients (HR 1.57, 95\% CI: 1.10-2.06, $\mathrm{P}=0.01$ ) (Table 3). In addition, male gender and a prior diagnosis of pneumonia were also associated with poorer survival in the multivariate analysis (Table 3).

\section{Histology and survival}

We analyzed three different histologic subgroups "adenocarcinoma", which included adenocarcinoma in situ, adenocarcinoma with mixed subtype, adenocarcinoma 


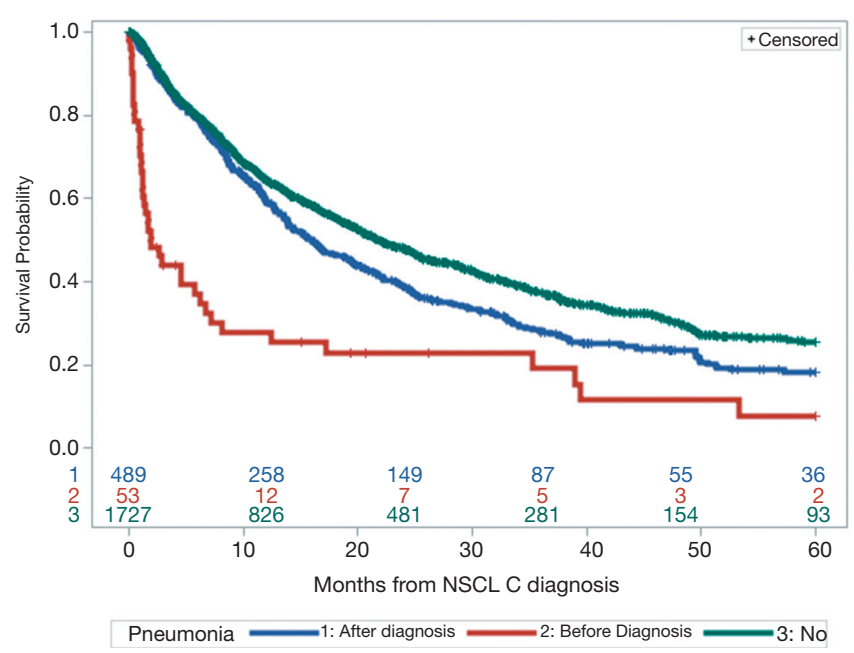

Figure 1 Overall survival by pneumonia status. Kaplan-Meier analysis of all patients separated into cohorts based on pneumonia diagnosis. Patients with a pneumonia diagnosis after lung cancer diagnosis (blue line), diagnosis of pneumonia prior to lung cancer diagnosis (red line) and patients diagnosed with lung cancer without a diagnosis of pneumonia (green line). Numbers represent those surviving at each annual time point for each of the cohorts. $\mathrm{P}<0.001$.

with neuroendocrine differentiation, adenocarcinoma NOS, adenosquamous carcinoma, bronchiolo-alveolar adenocarcinoma, mixed cell adenocarcinoma, mucinproducing adenocarcinoma, mucinous adenocarcinoma and papillary adenocarcinoma; "squamous cell carcinoma", which included papillary squamous cell carcinoma, squamous cell carcinoma, squamous cell carcinoma keratinizing, squamous cell carcinoma large cell nonkeratinizing, squamous cell carcinoma spindle cell; and "other" which included carcinoma NOS, carcinoma undifferentiated, large cell carcinoma, non-small cell carcinoma. Compared to patients with adenocarcinoma, patients with squamous cell or other histology had inferior median overall survival (Figure S1 and Table S1). However, there was no significant interaction between pneumonia diagnosis and histologic subtype on survival (Table 3).

\section{Pneumonia and survival}

We assessed patients who had a diagnosis of pneumonia during the study period. The overall incidence of pneumonia prior to the diagnosis of lung cancer was $2.3 \%$. The overall survival of patients who had pneumonia before their lung cancer diagnosis was compared to that of patients who had pneumonia after the diagnosis of lung cancer and to that of patients without a diagnosis of pneumonia. Patients with pneumonia prior to the diagnosis of lung cancer had a decreased overall survival as compared to either patients diagnosed with pneumonia after lung cancer diagnosis (1.9 vs. 15.9 months, $\mathrm{P}<0.001)$ or patients who did not have any pneumonia (1.9 vs. 21.8 months, $\mathrm{P}<0.001)$ (Figure 1).

Given these findings, we next looked at the overall survival of patients with pneumonia grouped by age. Among younger patients, median overall survival was 4.2 , 14.3 , and 23 months for those with a prior diagnosis of pneumonia, pneumonia after lung cancer diagnosis, and no pneumonia, respectively (Figure 2A). However, it should be noted only 2 younger patients had prior pneumonia. Among older patients, the median overall survival was 1.9, 16.2 and 21.8 months, respectively (Figure 2B). The association between pneumonia and overall survival was similar across the two age groups.

By univariate analysis, patients who had pneumonia prior to their lung cancer diagnosis had a higher risk of death than those with pneumonia after lung cancer diagnosis (HR 2.38, 95\% CI: $1.75-3.33$; $\mathrm{P}<0.001$ ) or no pneumonia (HR 2.98, 95\% CI: 2.18-4.08; $\mathrm{P}<0.001$ ) (Table 4).

We next looked at how pneumonia diagnosis and stage affected overall survival irrespective of age. Prior pneumonia diagnosis was not associated with overall survival in stage $\mathrm{I} /$ II patients (Table 5). In stage III patients with pneumonia prior to lung cancer, median survival was lower than in those with pneumonia after lung cancer diagnosis or those without pneumonia (1.5 vs. 24.1 vs. 34.6 months); however, there were only 8 patients in this stage cohort with pneumonia diagnosis prior to lung cancer diagnosis (Figure 3).

In stage IV patients with pneumonia prior to lung cancer, median survival was also lower than patients with pneumonia after diagnosis or those without pneumonia (1.2 vs. 7.9 vs. 10.5 months) (Figure 3).

In multivariate analysis, the effect of pneumonia on overall survival depended on stage $(\mathrm{P}=0.0051)$ after adjusting for age, gender, race and smoking status (Table 3). Patients with stage III or stage IV NSCLC with pneumonia prior to lung cancer had decreased survival compared to patients with pneumonia after diagnosis or those with no pneumonia (Table 3).

\section{Discussion}

The current study provides clinical and pathological 

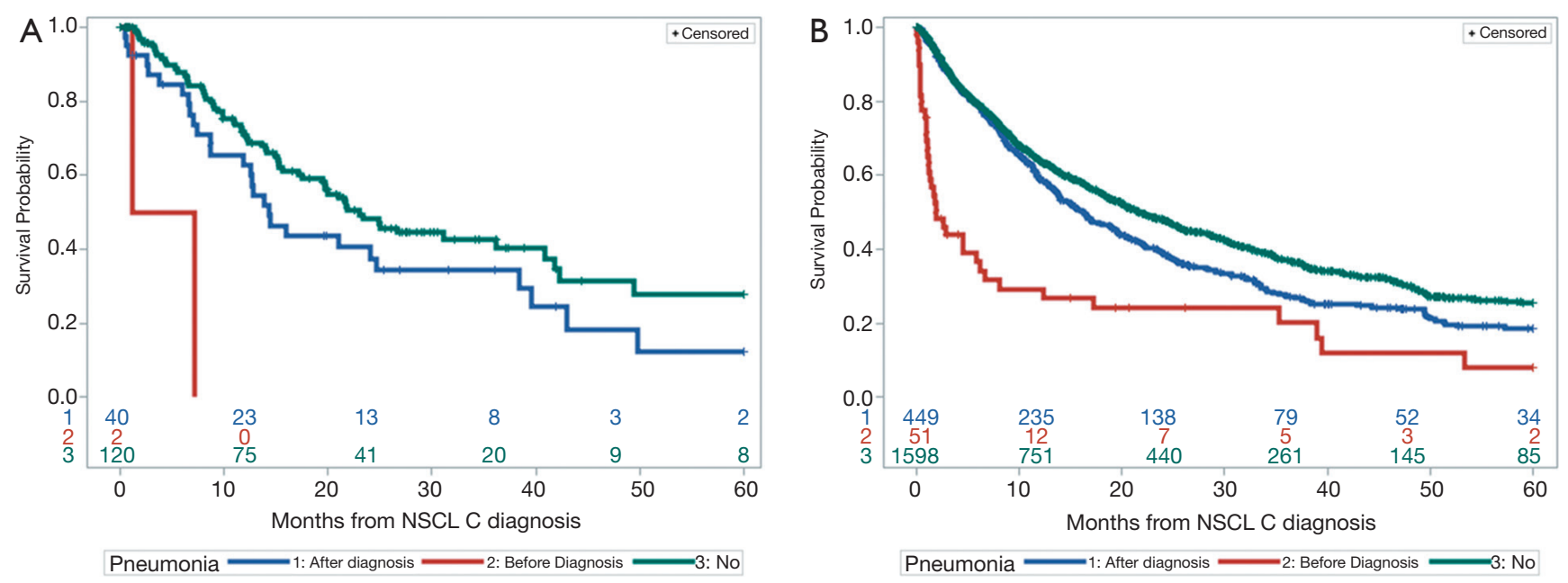

Figure 2 Overall survival by age group and pneumonia status. Kaplan-Meier analysis of (A) patients $\leq 50$ years of age stratified by pneumonia diagnosis and (B) patients $>50$ years of age stratified by pneumonia diagnosis.

Table 4 Pneumonia effects on overall survival stratified by age group

\begin{tabular}{|c|c|c|c|c|c|c|}
\hline Pneumonia & \multicolumn{2}{|c|}{ All patients } & \multicolumn{2}{|c|}{$\leq 50$ years } & \multicolumn{2}{|c|}{$>50$ years } \\
\hline Prior vs. after diagnosis & $2.38(1.75,3.33)$ & $<0.001$ & $6.77(1.56,29.4)$ & 0.01 & $2.34(1.68,3.27)$ & $<0.001$ \\
\hline Prior vs. no diagnosis & $2.98(2.18,4.08)$ & $<0.001$ & $9.89(2.34,41.89)$ & 0.002 & $2.86(2.07,3.94)$ & $<0.001$ \\
\hline
\end{tabular}

*Based on log-rank analysis of survival distribution.

Table 5 Pneumonia effects on overall survival stratified by stage

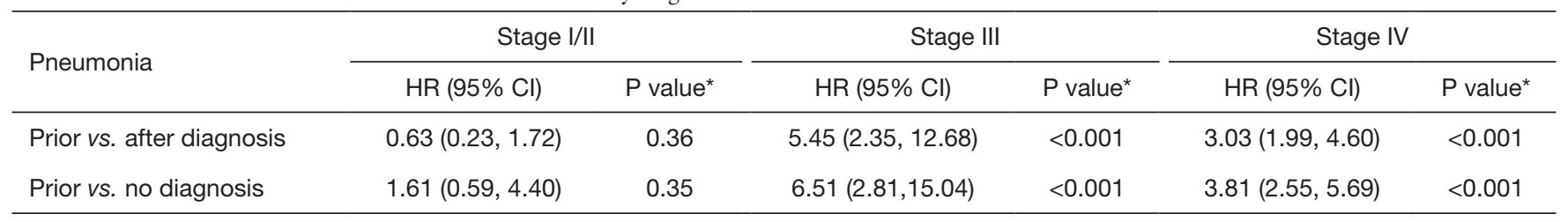

${ }^{*}$ Based on log-rank analysis of survival distribution.

data on NSCLC in younger versus older patients using a contemporaneous data set over the past decade. In the present retrospective study, $8 \%$ of patients seen at a single academic cancer center were 50 years of age or younger which is comparable to other studies $(10,19,22)$. Our data confirms and extends previous study results $(10,15,19)$ by demonstrating that younger patients had a higher likelihood of being never smokers, being diagnosed at a later stage of disease and having non-squamous histology than older patients. In addition, younger patients and female patients had an improved survival, confirming previously reported results $(10,11)$. Interestingly, having pneumonia prior to the diagnosis of lung cancer correlated with poorer survival across all age groups, particularly in patients with advancedstage lung cancers. The incidence of pneumonia prior to the diagnosis of lung cancer was $2.3 \%$, which is similar to that reported by Tang and colleagues, who found that the incidence of new lung cancer after pneumonia was approximately $1 \%$ within 90 days and $2 \%$ over 5 years. The authors concluded that routine chest radiographs after pneumonia were not warranted for detecting lung cancer. However, the study did suggest that patients $\geq 50$ years of age 

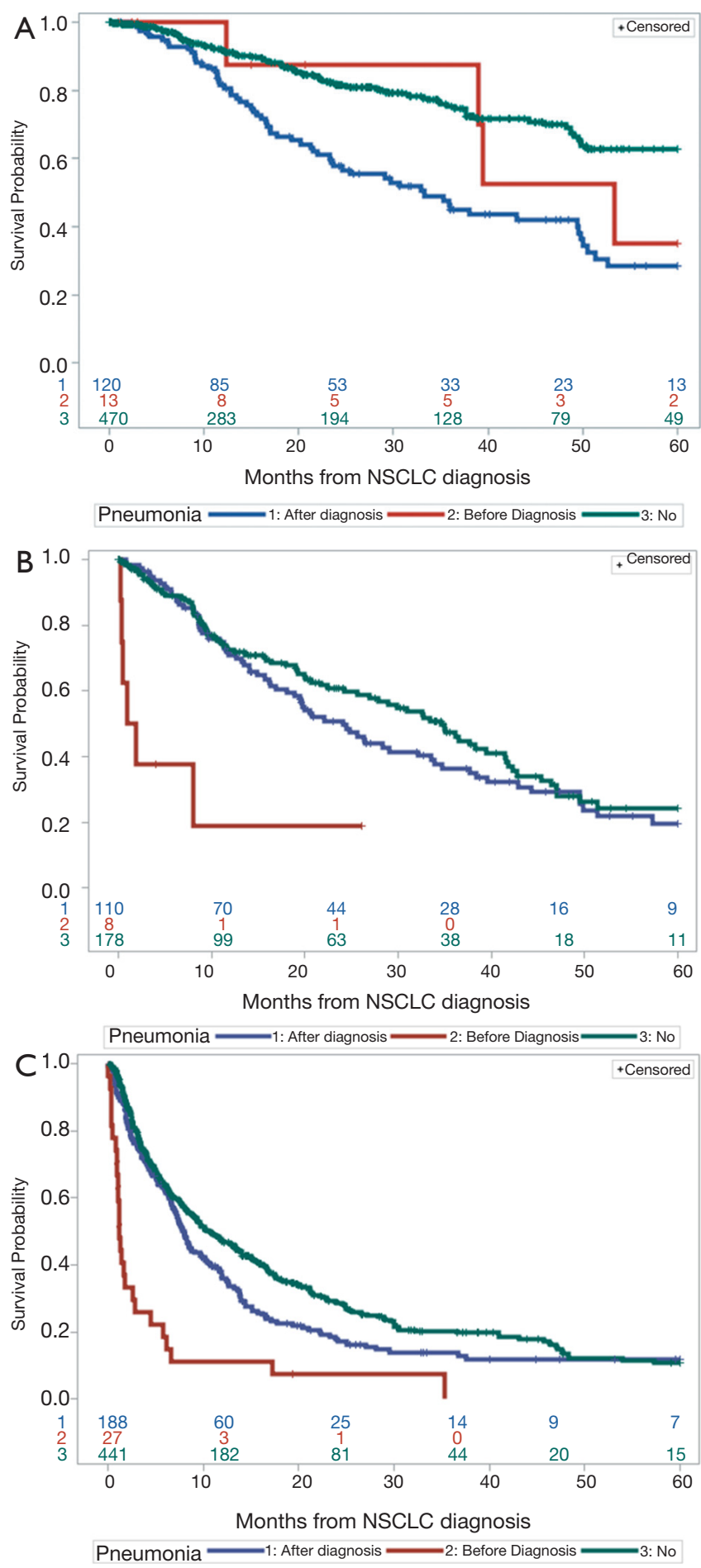

Figure 3 Overall survival by stage and pneumonia status. KaplanMeier analysis of (A) patients diagnosed with stage I/II NSCLC stratified by pneumonia diagnosis, (B) patients diagnosed with stage III NSCLC stratified by pneumonia diagnosis and (C) patients diagnosed with stage IV NSCLC stratified by pneumonia diagnosis. NSCLC, non-small cell lung cancer. should be targeted for radiographic follow-up (23). We found that patients with pneumonia prior to lung cancer diagnosis had worse overall survival than patients with pneumonia after lung cancer diagnosis or those without pneumonia. Guidelines from 2001 from the American Thoracic Society (ATS)/Infectious Diseases Society of America (IDSA) recommended repeat chest radiograph approximately 4 to 6 weeks after hospitalization for community acquired pneumonia (CAP) to exclude the possibility of malignancy associated CAP (Level III evidence) (24). However, this guideline was recently updated in 2019 and routine chest imaging following a diagnosis of CAP is now not recommended if symptoms resolve in 5-7 days (25). One of the limitations of the current study is the small number of young patients with pneumonia diagnosed prior to cancer diagnosis, so larger studies would be warranted to assess whether post-pneumonia chest CT scans would be of benefit in this population.

Our data also demonstrate that prior diagnosis of pneumonia in advanced-stage NSCLC is associated with decreased median overall survival. This may be due to increased tumor burden and larger lung masses that would predispose for obstructive pneumonia. Alternatively, pneumonia may have effects on the immune and tumor microenvironment that promotes tumorigenesis or cancer progression. This effect could be mediated by the local lung microbiome and/or inflammation. Multiple studies have demonstrated that development of lung cancer is strongly associated with inflammation (26). Recent studies have also suggested that the lung commensal microbiome may play a role in the development and/or progression of lung cancer through resident-lung T-cells (27). Furthermore, these resident-lung immune cells have been demonstrated to be important in maintaining pulmonary homeostasis and providing immune surveillance in response to invading pathogens (28). Certain infections such as, Chlamydia pneumonia, have been linked to the development of lung cancer (29). In addition, a recent paper suggested that there is enrichment of the Streptococcus genus within the lungs of patients with lung cancer as compared to healthy patients or patients with other lung disease (30). Therefore, it is possible that certain microorganisms or dysbiosis of the lung microbiota could disrupt the homeostatic immune function in the lung allowing for the development or progression of lung cancer. Future studies could determine which specific bacterial, viral, fungal or parasitic organisms infected those lung cancer patients with pneumonia pre-dating their lung cancer diagnosis. 
Our study had several limitations. First, the number of younger patients was significantly smaller than the number of older patients. Similarly, analysis of patients with pneumonia was also limited by sample size, especially in the younger patient cohort, which decreases the reliability of the results. In the future, larger multi-site studies or meta-analysis will be needed to more fully test the general applicability of these data. Second, data collection and analysis were retrospective and confined to one institution therefore, making it harder to apply our results broadly to other institutions. Third, data on mutational analysis and treatment modalities were not uniform, and therefore, were not collected. Finally, details regarding the specifics of pneumonia (method of diagnosis, microbiology and treatment) were not uniformly available.

\section{Conclusions}

Despite the limitations of the study, our findings confirm the characterization of young patients with lung cancer and further expand knowledge regarding the potential importance of pneumonia in both younger and older patients with lung cancer. These data suggest that care should be taken in patients with pneumonia to ensure that there is no underlying malignancy. Given patients diagnosed with pneumonia prior to lung cancer diagnosis had poorer clinical outcomes further studies addressing underlying etiology leading to pneumonia as well as radiologic characterization of these patients could potentially lead to clinical interventions that would improve outcomes. Further study of the role pneumonia may play in the disruption of the lung microbiota and pulmonary immune homeostasis as it relates to lung cancer may have a significant impact on how we understand the genesis and progression of lung cancer. Such research could lead to novel therapeutic targets and potentially better treatment and disease outcomes in lung cancer patients.

\section{Acknowledgments}

Funding: None.

\section{Footnote}

Reporting Checklist: The authors have completed the STROBE reporting checklist. Available at http://dx.doi. org/10.21037/jtd-20-2891

Data Sharing Statement: Available at http://dx.doi. org/10.21037/jtd-20-2891

Peer Review File: Available at http://dx.doi.org/10.21037/jtd20-2891

Conflicts of Interest: All authors have completed the ICMJE uniform disclosure form (available at http://dx.doi. org/10.21037/jtd-20-2891). The authors have no conflicts of interest to declare.

Ethical Statement: The authors are accountable for all aspects of the work in ensuring that questions related to the accuracy or integrity of any part of the work are appropriately investigated and resolved. The study was conducted in accordance with the Declaration of Helsinki (as revised in 2013). The study was approved by the University of Michigan Institutional Review Board (IRB) (HUM00125235) and individual consent for this retrospective analysis was waived.

Open Access Statement: This is an Open Access article distributed in accordance with the Creative Commons Attribution-NonCommercial-NoDerivs 4.0 International License (CC BY-NC-ND 4.0), which permits the noncommercial replication and distribution of the article with the strict proviso that no changes or edits are made and the original work is properly cited (including links to both the formal publication through the relevant DOI and the license). See: https://creativecommons.org/licenses/by-nc-nd/4.0/.

\section{References}

1. Moolgavkar SH, Holford TR, Levy DT, et al. Impact of reduced tobacco smoking on lung cancer mortality in the United States during 1975-2000. J Natl Cancer Inst 2012;104:541-8.

2. Siegel RL, Miller KD, Jemal A. Cancer statistics, 2020. CA Cancer J Clin 2020;70:7-30.

3. Boffetta $P$, Jarvholm B, Brennan P, et al. Incidence of lung cancer in a large cohort of non-smoking men from Sweden. Int J Cancer 2001;94:591-3.

4. Jemal A, Miller KD, Ma J, et al. Higher Lung Cancer Incidence in Young Women Than Young Men in the United States. N Engl J Med 2018;378:1999-2009.

5. Zhong YJ, Wen YF, Wong HM, et al. Trends and Patterns of Disparities in Burden of Lung Cancer in the United States, 1974-2015. Front Oncol 2019;9:404.

6. Forastiere F, Perucci CA, Arca M, et al. Indirect estimates 
of lung cancer death rates in Italy not attributable to active smoking. Epidemiology 1993;4:502-10.

7. Enstrom JE. Rising lung cancer mortality among nonsmokers. J Natl Cancer Inst 1979;62:755-60.

8. Thun MJ, Hannan LM, Adams-Campbell LL, et al. Lung cancer occurrence in never-smokers: an analysis of 13 cohorts and 22 cancer registry studies. PLoS Med 2008;5:e185.

9. Thun MJ, Henley SJ, Burns D, et al. Lung cancer death rates in lifelong nonsmokers. J Natl Cancer Inst 2006;98:691-9.

10. Gadgeel SM, Ramalingam S, Cummings G, et al. Lung cancer in patients $<50$ years of age: the experience of an academic multidisciplinary program. Chest 1999;115:1232-6.

11. Ramalingam S, Pawlish K, Gadgeel S, et al. Lung cancer in young patients: analysis of a Surveillance, Epidemiology, and End Results database. J Clin Oncol 1998;16:651-7.

12. Owonikoko TK, Ragin CC, Belani CP, et al. Lung cancer in elderly patients: an analysis of the surveillance, epidemiology, and end results database. J Clin Oncol 2007;25:5570-7.

13. Bryant AS, Cerfolio RJ. Differences in outcomes between younger and older patients with non-small cell lung cancer. Ann Thorac Surg 2008;85:1735-9; discussion 1739.

14. Tas F, Ciftci R, Kilic L, et al. Age is a prognostic factor affecting survival in lung cancer patients. Oncol Lett 2013;6:1507-13.

15. Subramanian J, Morgensztern D, Goodgame B, et al. Distinctive characteristics of non-small cell lung cancer (NSCLC) in the young: a surveillance, epidemiology, and end results (SEER) analysis. J Thorac Oncol 2010;5:23-8.

16. Thomas A, Chen Y, Yu T, et al. Trends and Characteristics of Young Non-Small Cell Lung Cancer Patients in the United States. Front Oncol 2015;5:113.

17. Arnold BN, Thomas DC, Rosen JE, et al. Lung Cancer in the Very Young: Treatment and Survival in the National Cancer Data Base. J Thorac Oncol 2016;11:1121-31.

18. Ak G, Metintas M, Metintas S, et al. Lung cancer in individuals less than 50 years of age. Lung 2007;185:279-86.

19. Sacher AG, Dahlberg SE, Heng J, et al. Association Between Younger Age and Targetable Genomic Alterations and Prognosis in Non-Small-Cell Lung Cancer. JAMA Oncol 2016;2:313-20.

20. Radzikowska E, Roszkowski K, Glaz P. Lung cancer in patients under 50 years old. Lung Cancer 2001;33:203-11.

21. Lara MS, Brunson A, Wun T, et al. Predictors of survival for younger patients less than 50 years of age with nonsmall cell lung cancer (NSCLC): a California Cancer Registry analysis. Lung Cancer 2014;85:264-9.

22. Suidan AM, Roisman L, Belilovski Rozenblum A, et al. Lung Cancer in Young Patients: Higher Rate of Driver Mutations and Brain Involvement, but Better Survival. J Glob Oncol 2019;5:1-8.

23. Tang KL, Eurich DT, Minhas-Sandhu JK, et al. Incidence, correlates, and chest radiographic yield of new lung cancer diagnosis in 3398 patients with pneumonia. Arch Intern Med 2011;171:1193-8.

24. Niederman MS, Mandell LA, Anzueto A, et al. Guidelines for the management of adults with communityacquired pneumonia. Diagnosis, assessment of severity, antimicrobial therapy, and prevention. Am J Respir Crit Care Med 2001;163:1730-54.

25. Metlay JP, Waterer GW, Long AC, et al. Diagnosis and Treatment of Adults with Community-acquired Pneumonia. An Official Clinical Practice Guideline of the American Thoracic Society and Infectious Diseases Society of America. Am J Respir Crit Care Med 2019;200:e45-67.

26. Palucka AK, Coussens LM. The Basis of Oncoimmunology. Cell 2016;164:1233-47.

27. Jin C, Lagoudas GK, Zhao C, et al. Commensal Microbiota Promote Lung Cancer Development via gammadelta T Cells. Cell 2019;176:998-1013.e16.

28. Lloyd CM, Marsland BJ. Lung Homeostasis: Influence of Age, Microbes, and the Immune System. Immunity 2017;46:549-61.

29. Littman AJ, Jackson LA, Vaughan TL. Chlamydia pneumoniae and lung cancer: epidemiologic evidence. Cancer Epidemiol Biomarkers Prev 2005;14:773-8.

30. Tsay JJ, Wu BG, Badri MH, et al. Airway Microbiota Is Associated with Upregulation of the PI3K Pathway in Lung Cancer. Am J Respir Crit Care Med 2018;198:1188-98.

Cite this article as: Weinberg FD, Zhao L, Chellappa N, Kalemkerian GP, Ramnath N. Characterization of pneumonia and other factors leading to poorer survival across all age groups in patients with non-small cell lung cancer (NSCLC). J Thorac Dis 2021;13(2):986-994. doi: 10.21037/jtd-20-2891 


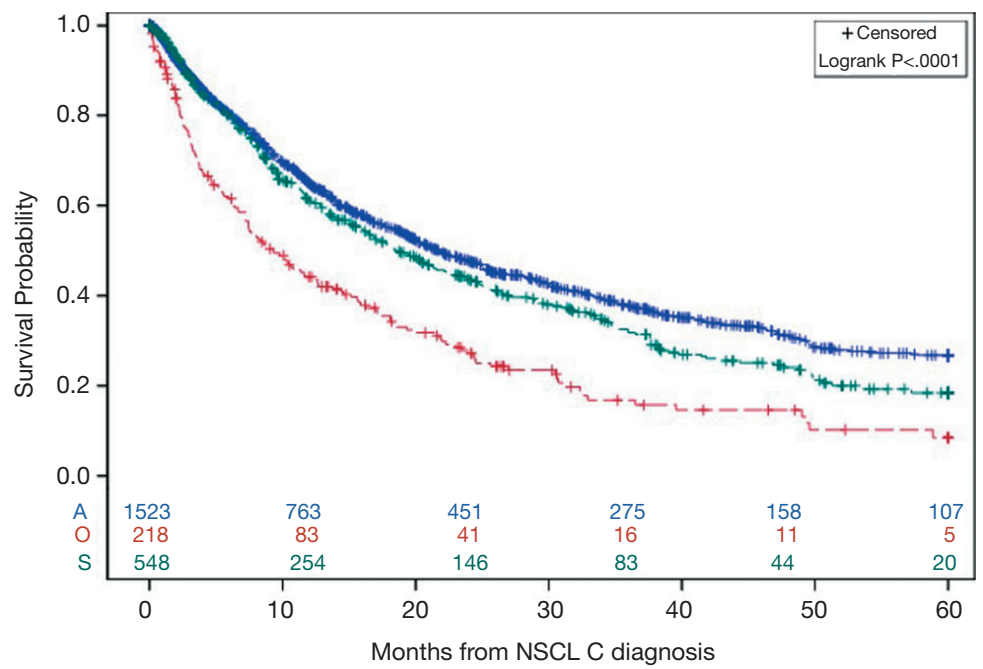

Figure S1 Overall survival by histologic subgroup. Kaplan-Meier analysis of all patients separated into cohorts based on histology. Patients categorized as adenocarcinoma (A, blue line) included adenocarcinoma in situ, adenocarcinoma with mixed subtype, adenocarcinoma with neuroendocrine differentiation, adenocarcinoma NOS, adenosquamous carcinoma, bronchiolo-alveolar adenocarcinoma, mixed cell adenocarcinoma, mucin-producing adenocarcinoma, mucinous adenocarcinoma and papillary adenocarcinoma; squamous cell carcinoma (S, green line) included papillary squamous cell carcinoma, squamous cell carcinoma, squamous cell carcinoma keratinizing, squamous cell carcinoma large cell nonkeratinizing, squamous cell carcinoma spindle cell; and other (O, red line) included carcinoma NOS, carcinoma undifferentiated, large cell carcinoma, non-small cell carcinoma. Numbers represent those surviving at each annual time point for each of the cohorts. $\mathrm{P}<0.001$.

Table S1 Median survival according to histology subtype

\begin{tabular}{lcc}
\hline Histology & Median Survival (months) & 95\% Confidence Interval \\
\hline Adenocarcinoma & 21.8 & $19.8-24.7$ \\
Squamous cell carcinoma & 18.5 & $15.9-22.3$ \\
Other & 9.3 & $7.2-12.6$ \\
\hline
\end{tabular}

